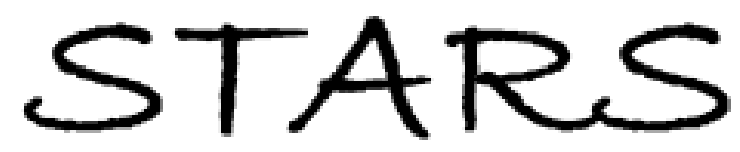

University of Central Florida

STARS

$1-1-2011$

\title{
Fabrication of Aligned Carbon Nanotube Array Electrodes for Organic Electronic Devices
}

\author{
Biddut K. Sarker \\ University of Central Florida \\ Muhammad R. Islam \\ University of Central Florida \\ Feras Alzubi \\ University of Central Florida \\ Saiful I. Khondaker \\ University of Central Florida
}

Find similar works at: https://stars.library.ucf.edu/facultybib2010

University of Central Florida Libraries http://library.ucf.edu

This Article is brought to you for free and open access by the Faculty Bibliography at STARS. It has been accepted for inclusion in Faculty Bibliography 2010 s by an authorized administrator of STARS. For more information, please contactSTARS@ucf.edu.

\section{Recommended Citation}

Sarker, Biddut K.; Islam, Muhammad R.; Alzubi, Feras; and Khondaker, Saiful I., "Fabrication of Aligned Carbon Nanotube Array Electrodes for Organic Electronic Devices" (2011). Faculty Bibliography 2010s. 1854.

https://stars.library.ucf.edu/facultybib2010/1854

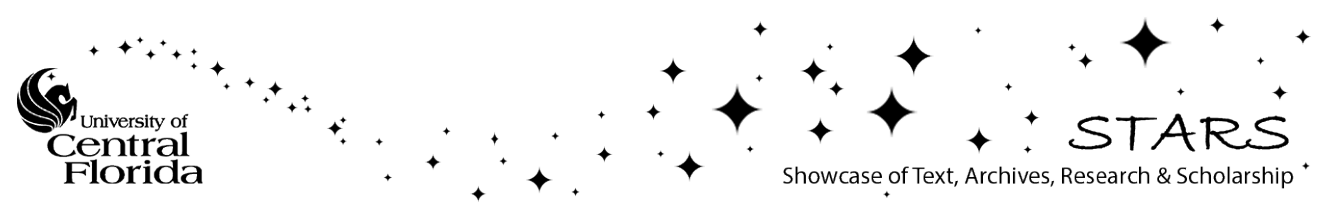




\title{
Fabrication of Aligned Carbon Nanotube Array Electrodes for Organic Electronic Devices
}

\author{
Biddut K. Sarker ${ }^{1,2}$, Muhammad R. Islam ${ }^{1,2}$, Feras Alzubi ${ }^{1,2}$, and Saiful I. Khondaker 1,2,3,* \\ ${ }^{1}$ Nanoscience Technology Center, ${ }^{2}$ Department of Physics, and ${ }^{3}$ School of Electrical Engineering and Computer Science, \\ University of Central Florida, 12424 Research Parkway, suite 400, Orlando, Florida 32826, USA
}

We report fabrication of aligned array carbon nanotube (CNT) electrodes for organic electronic device applications. The ultra-high density aligned CNT array was assembled via dielectrophoresis (DEP) from a high quality surfactant free and stable aqueous CNT solution. The CNT source and drain electrodes were formed though oxidative cutting of aligned CNT array by electron beam lithography and precise oxygen plasma etching. As an example of usefulness of this electrode, organic field effect transistor was fabricated using a thin film of poly (3-hexylthiophene) and the electrical transport properties of the devices were studied. Compared to the control devices fabricated with gold electrode, devices based on CNT array electrode show better performance with higher mobility, higher current on/off ratio and higher reproducibility. This study demonstrates that aligned array CNT electrode will have potential applications in fabricating organic electronic devices.

Keywords: Carbon Nanotubes, Organic Transistors, Charge Transport, Electrodes, Dielectrophoresis, Interface, Contact Barrier.

Organic field effect transistor (OFET) has attracted tremendous attention due to their low-cost fabrication process and prospect for large area, flexible, transparent electronic device applications. ${ }^{1,2}$ The performance of the OFET device is not only depends on the molecular properties of the organic semiconducting material, but also it

\footnotetext{
*Author to whom correspondence should be addressed. Email: saiful@mail.ucf.edu
}

is extremely sensitive to the organic material-metal electrode interface. ${ }^{4,5}$ The metals such as gold, palladium are widely used as source and drain (S/D) electrodes for OFET device. ${ }^{3}$ When metal electrodes are contacted with organic semiconducting material, in addition to the Schottky barrier, dipole barrier is formed at the interface. ${ }^{6,7}$ As a result, the contact resistance between the metal and semiconductor become very large and charge transport is often limited by the interface. ${ }^{1,4,5-9}$ In addition, metal electrodes are not solution processed and they are not flexible. However, flexible electrodes are required in the application of flexible electronics.

In order to overcome the problems of high contact resistance, dipole formation and non flexibility of metal electrodes, carbon nanotube (CNT) has been considered as a promising electrode materials for organic electronics due to their highly conducting, flexible, and one-dimensional structure. ${ }^{10-12}$ Individual CNTs has been used as an electrode material in contacting single molecule or making nanoscale OFET using pentacene ${ }^{12-14}$ and was found to be an efficient charge injector. The reason for such an enhancement is that when an electric field is applied in CNT electrodes, a large local field is generated at the nanotube apex and increases charge injection. ${ }^{15,16}$ However individual CNT electrodes are not appropriate for practical application in large area electronic devices.

Several approaches have been reported for the fabrication of large area OFETs using carbon nanotube electrodes ${ }^{15-19}$ all of which showed improved device performance compared to the control metal electrode OFET devices. The most commonly used approach is to use random network of CNTs as electrode materials. ${ }^{17-18}$ However, it was noted that optimum charge injection may not occur due to the random alignment of CNT tips. OFET devices were also fabricated using CNT/polymer 
composite as an electrode material. ${ }^{19}$ One possible drawback of the CNT/polymer composite electrodes is that when gate voltage is applied to the OFET, a part of the applied gate voltage also modulates the electrodes which affect the device performance. In another report, CNT array electrode was fabricated by anchoring CNTs onto titanium contact using vacuum filtration method. ${ }^{15,16}$ In this electrode pattern, CNTs appears to be random, density of the CNT is very low and dimension of the electrodes appears to be not well defined which may cause charge injection not only from the CNT electrode but also from the base metal electrode where CNTs are anchored. Therefore, more efficient charge injection may be obtained if CNT electrode is fabricated from ultra-high density aligned array where individual CNTs are closely packed with tips parallel to each others with well defined channel lengths.

Here, we demonstrated a novel, controlled and highly reproducible approach for the fabrication of CNT aligned array electrode. The ultra-high density CNTs were aligned with a very high linear density $(\sim 30 \mathrm{CNTs} / \mu \mathrm{m})$ from high quality CNT aqueous solution by dielectrophoresis techniques. The CNT source and drain electrodes are fabricated with electron beam lithography (EBL) followed by etching CNTs with oxygen plasma. In order to examine the functionality and efficiency of the CNT array electrodes, we fabricated P3HT thin film OFET and compared the device performance with control gold electrode OFET of same dimension and fabricated under identical condition. Electrical transport measurements show that the device with CNT electrode has a higher on/off ratio and higher mobility compared to control OFET with gold electrode.

A schematic of the aligned CNT array electrode fabrication process is shown in Figure 1. An important step of the CNT array electrode fabrication is the alignment of CNT with ultra-high density and was done via dielectrophoretic (DEP) technique from a high quality aqueous solution of CNT. Using DEP, CNT can be directly integrated to the prefabricated patterns in a $2 \mathrm{D}$ array with controllable alignment and linear density. ${ }^{20}$ DEP has been shown to assemble $2 D, 1 D$ and $O D$ nanomaterials at the selected position of the circuit for device applications. ${ }^{21-25}$ Figure 1(a) shows a cartoon of the DEP set up for CNT assembly. Details of the CNT alignment and characterization can be found in our recent publication. ${ }^{20}$ In brief,

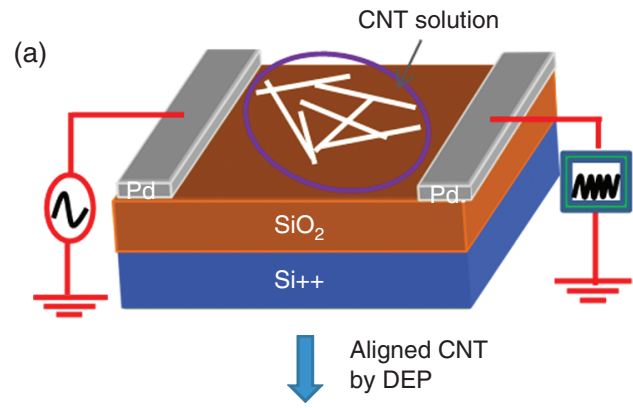

(b)

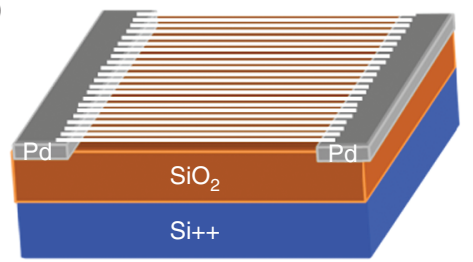

(c)

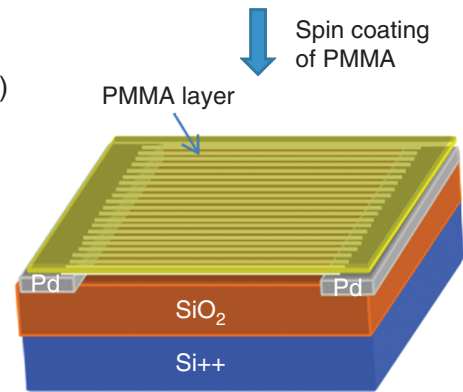

(f)

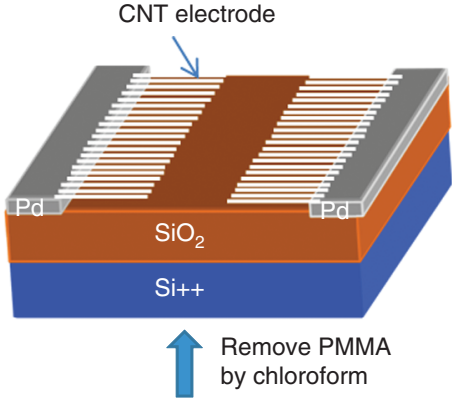

(e)

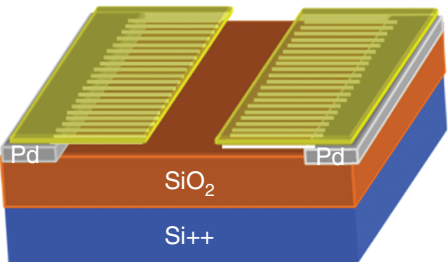

Etching CNT by $\mathrm{O}_{2}$ plasma

(d)

Open window by EBL

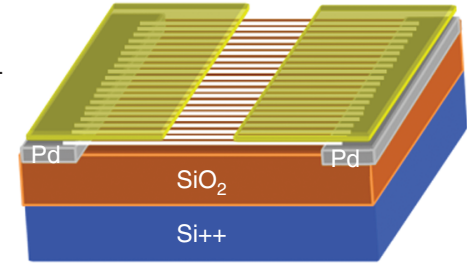

Fig. 1. Schematic illustration of carbon nanotube aligned array electrodes fabrication method. (a, b) aligning ultra-high density SWNTs from high quality CNT aqueous solution via DEP between prefabricated palladium (Pd) patterns, (c) spin coating of PMMA resist, (d) opening an window on the CNTs array via electron beam lithography, (e) etching the exposed SWNTs by oxygen plasma and finally (f) removing PMMA using chloroform. 
palladium $(\mathrm{Pd})$ were used as a base material to anchor the aligned CNTs as Pd is known to make good electrical contact to CNTs. ${ }^{26} \mathrm{Pd}$ patterns of $5 \mu \mathrm{m} \times 25 \mu \mathrm{m}$ were fabricated on heavily doped silicon substrates capped with a thermally grown $250 \mathrm{~nm}$ thick $\mathrm{SiO}_{2}$ layer using standard EBL followed by deposition of $\mathrm{Cr}(3 \mathrm{~nm})$ and $\mathrm{Pd}(27 \mathrm{~nm})$ and standard lift off.

Highly purified, stable and surfactant free single walled CNT aqueous solution obtained from Brewer Science $\operatorname{Inc}^{27}$ and was diluted by six times in DI water. The solution was free from surfactant, catalytic particles and bundles, and contained mostly individual CNTs. The average diameter of the CNTs was $1.7 \mathrm{~nm}$ and the length of the nanotubes varied from 0.3 to $10 \mu \mathrm{m}$ with a median value of $1.5 \mu \mathrm{m}$ as determined from atomic force microscopy (AFM) and scanning electron microscopy (SEM) investigations. ${ }^{20}$ A $3 \mu \mathrm{L}$ drop of the solution was placed on the Pd patterns and an ac voltage of $5 \mathrm{Vp}-\mathrm{p}$ at $300 \mathrm{kHz}$ was applied between the Pd patterns for 30 seconds (Fig. 1(a)). The ac voltage gives rise to a time averaged DEP force $\left(F_{\mathrm{DEP}}\right)$ which for an elongated object is given by

$$
\begin{gathered}
F_{\mathrm{DEP}} \propto \varepsilon_{m} \operatorname{Re}\left[K_{f}\right] \nabla E_{\mathrm{RMS}}^{2} ; \quad K_{f}=\frac{\varepsilon_{p}^{*}-\varepsilon_{m}^{*}}{\varepsilon_{m}^{*}}, \\
\varepsilon_{p, m}^{*}=\varepsilon_{p, m}-i \frac{\sigma_{p, m}}{\omega}
\end{gathered}
$$

where $\varepsilon_{p}$ and $\varepsilon_{m}$ are the permittivity of the nanotube and solvent respectively, $K_{f}$ is the Claussius-Mossotti factor, $\sigma$ is the conductivity, and $\omega=2 \pi f$ is the frequency of the applied ac voltage. ${ }^{28}$ The induced dipole moments of the nanotube interacting with the strong electric field makes the nanotubes to move in a translational motion along the electric field gradient and align the nanotubes in the direction of the electric field lines (Fig. 1(b)). After the CNT assembly, PMMA was spin coated on the samples at $4000 \mathrm{rpm}$ for 60 seconds followed by baking at $180{ }^{\circ} \mathrm{C}$ for 15 minutes on a hotplate (Fig. 1(c)). The CNT electrode pattern of channel length $L=2 \mu \mathrm{m}$ and width $W=25 \mu \mathrm{m}$ was then defined by opening a window in PMMA resist via EBL writing and developing in a mixture of MIBK and IPA. The samples were then placed in a oxygen plasma chamber and exposed CNTs were etched away through the open window (Fig. 1(d)). Finally, devices were kept into the chloroform for 12 hours and rinse with acetone, IPA, and DI water to remove the remaining PMMA (Fig. 1(e)). Gold $(\mathrm{Au})$ electrodes of identical dimension were also fabricated using EBL for control experiments.

P3HT powder was purchased from EMD chemicals Inc. and was dissolved into 1,2 dichlorobenzene by stirring the solution with magnetic stirrer for 2 hours at $40{ }^{\circ} \mathrm{C}$. The concentration of the solution was $5 \mathrm{mg} / \mathrm{ml}$. The solution was then filtered with $0.2 \mu \mathrm{m}$ syringe filter. A thin film $(\sim 80 \mathrm{~nm})$ of $\mathrm{P} 3 \mathrm{HT}$ was then deposited onto chips containing both CNT and Au electrode by drop cast method inside a $\mathrm{N}_{2}$ glove box. The fabricated devices were then thermally annealed at $130-140{ }^{\circ} \mathrm{C}$ on a hot plate for 15 minutes to evaporate the solvent. The electrical transport measurements of the CNT arrays was carried out by DL instruments 1211 current preamplifier and a Keithdey 2400 source meter interfaced with LabView program. The OFET characteristics were measured using HewlettPacked (HP) 4145B semiconductor parametric analyzer connected to a probe station inside an enclosed glove box system filled with nitrogen gas.
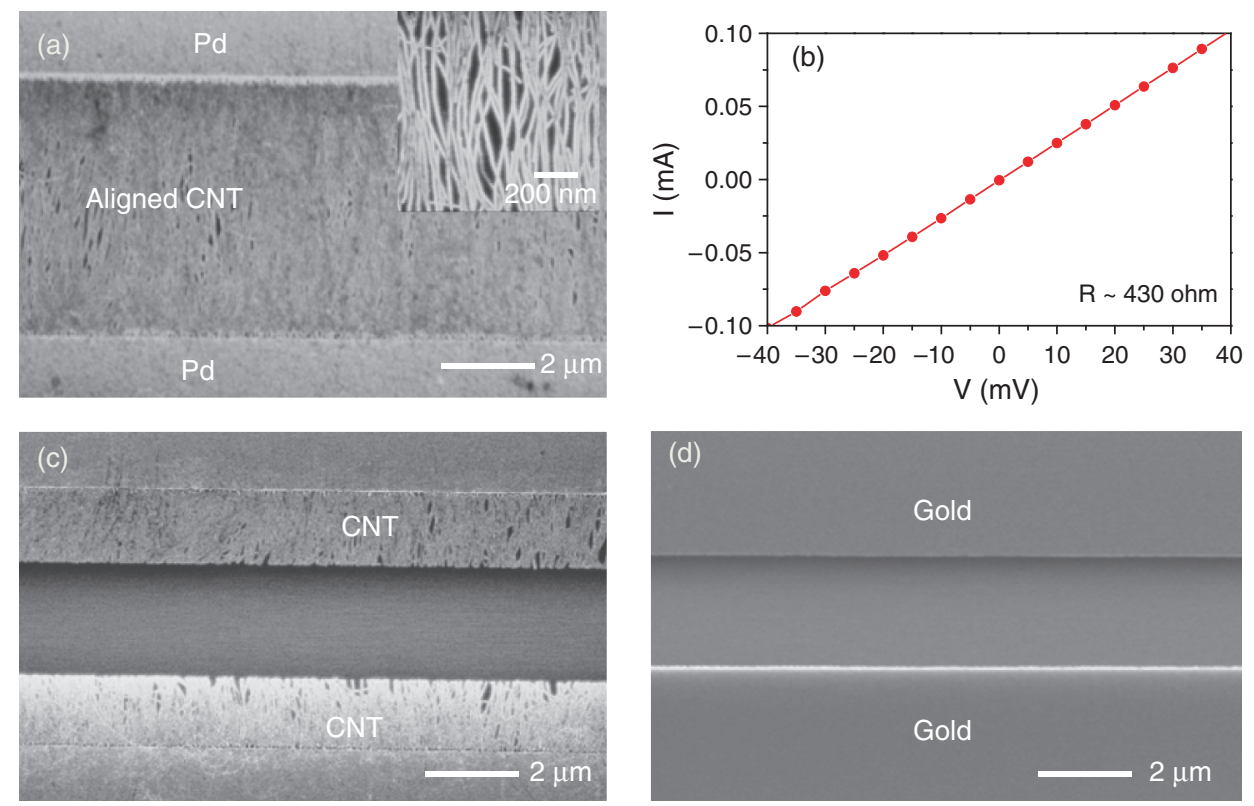

Fig. 2. (a) Scanning electron microscopy (SEM) image of a densely aligned CNT array. Inset: High magnification SEM image. (b) Current (I)-voltage $(V)$ characteristics of the aligned array. (c) SEM images of the CNT electrode after oxidative cutting and (d) gold electrode for control experiment. 
Figure 2(a) shows a representative SEM image of a densely aligned array of CNT assembled via DEP. Inset shows a high magnification SEM image of a part of the array. We have recently shown that by controlling the concentration of the CNT in the solution, we can reproducibly control the linear density of the CNT in the array from $1 \mathrm{CNT} / \mu \mathrm{m}$ to $30 \mathrm{CNT} / \mu \mathrm{m} .{ }^{20}$ This study also showed that at low densities, almost all of the nanotubes are well aligned and are parallel to each other whereas, at higher density, about $90 \%$ are aligned within $\pm 10^{\circ}$ of the longitudinal axis. ${ }^{20}$ In this experiment, we only used densely packed aligned array with a density of $\sim 30 \mathrm{CNT} / \mu \mathrm{m}$ so that the resistance of the array is low $(<500 \Omega)$. Figure 2(b) shows the current-voltage characteristics of the array from which we calculate a resistance of $\sim 430 \Omega$ with a corresponding resistivity of $\sim 2 \mathrm{k} \Omega / \mathrm{sq}$. In addition we have also verified the metallic nature of the array by measuring current-gate voltage characteristics (not shown here). The low resistance and metallic behavior of the array makes them ideal material for OFET electrodes.

Figure 2(c) shows a SEM image of our fabricated CNT electrodes after oxidative cutting with well defined channel length and width. One fundamental advantage of our electrode pattern is that all the tips of CNTs in the electrodes are parallel and open ended; because in our experiment, electrodes are formed though oxidative cutting of each CNTs in the middle by oxygen plasma etching. This is in distinct contrast to the previous reports of CNT electrode fabrication. The parallel and open ended tips of our electrode design are advantageous because CNT electrodes tunnels the charge carrier though the contact barrier due to electric field enhancement and the field emission is larger for the open CNT than the close end CNT. ${ }^{15,29}$

As an example of the effectiveness of our aligned CNT array electrode, we fabricated P3HT thin film OFET using CNT array electrodes (CNT/OFET). For control experiment, OFET with gold electrodes was also fabricated (Au/OFET). The SEM of the gold electrode is shown in Figure 2(d). The source-drain current $\left(I_{\mathrm{d}}\right)$ versus sourcedrain voltage $\left(V_{\mathrm{d}}\right)$ characteristics (output curve) of the CNT/OFET and Au/OFET were measured at different gate voltages $\left(V_{\mathrm{g}}\right)$ and are shown in Figures 3(a) and (b) respectively. Compared to the output curve of Au/OFET device, output curve of CNT/OFET device shows better drain current modulation with $V_{\mathrm{g}}$. In addition, saturation current $I_{d \text {, sat }}(2.5 \mu \mathrm{A})$ of CNT/OFET device is higher than the saturation current $(0.9 \mu \mathrm{A})$ of the Au/OFET device at $V_{\mathrm{g}}=$ $-80 \mathrm{~V}$. In low $V_{\mathrm{ds}}$ region, the curve shows a sub-linear onset due to contact resistance, hallmark of short channel OFETs. Since the channel length of our device is only $2 \mu \mathrm{m}$, the nonlinear onset behavior appears in our device. Similar nonlinear behavior has also been observed for the OFETs fabricated with RGO electrode ${ }^{30}$ as well as OFET with metal electrode. ${ }^{31}$

Transfer characteristics of the CNT/OFET and $\mathrm{Au} / \mathrm{OFET}$ device are shown in left axis in Figures 4(a)
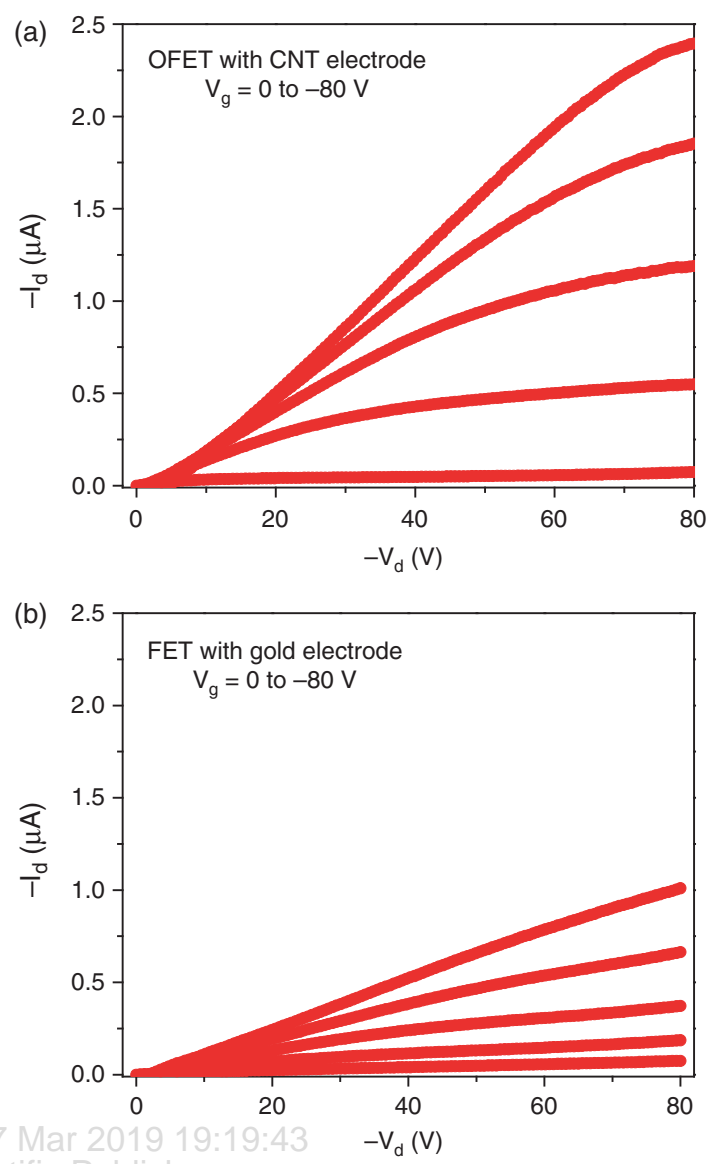

Fig. 3. Output characteristics ( $I_{\mathrm{d}}$ vs. $V_{\mathrm{d}}$ curve) of P3HT OFET at various $V_{\mathrm{g}}$ for (a) CNT aligned array electrodes and (b) gold electrode. $V_{\mathrm{g}}=0,-20,-40,-60$ and $-80 \mathrm{~V}$ from bottom to top. $L=2 \mu \mathrm{m}$ and $W=25 \mu \mathrm{m}$. Both curves are plotted in the same scale to see the clear difference in output current for these two OFETs.

and (b) respectively, where $I_{\mathrm{d}}$ is plotted as a function of $V_{\mathrm{g}}$ with a fixed $V_{\mathrm{d}}=-60 \mathrm{~V}$. The $\sqrt{ } I_{d}$ versus $V_{\mathrm{g}}$ plots are shown right axis of Figures 4(a) and (b), from where threshold voltage $\left(V_{\mathrm{T}}\right)$ was calculated to be $0 \mathrm{~V}$ and $10 \mathrm{~V}$ for CNT/OFET and Au/OFET device respectively. From here, the saturation mobility $\left(\mu_{\text {sat }}\right)$ and linear mobility $\left(\mu_{\text {lin }}\right)$ of the devices were calculated using the standard formula $\mu_{\text {sat }}=I_{d \text {, sat }}\left(2 L / W C_{i}\right)\left(1 /\left(V_{\mathrm{g}}-V_{\mathrm{T}}\right)^{2}\right)$ and $\mu_{\text {lin }}=$ $\left(L / W C_{i} V_{\mathrm{d}}\right)\left(d I_{\mathrm{d}} / d V_{\mathrm{g}}\right)$ respectively; where $C_{i}$ is the capacitance per unit area of the gate insulator $\left(13.8 \mathrm{nF} / \mathrm{cm}^{2}\right){ }^{32}$ The calculated $\mu_{\text {lin }}$ and $\mu_{\text {sat }}$ of CNT/OFET device are 0.004 and $0.005 \mathrm{~cm}^{2} / \mathrm{Vs}$ respectively. On the other hand, both $\mu_{\text {lin }}$ and $\mu_{\text {sat }}$ of Au/OFET device are $0.001 \mathrm{~cm}^{2} / \mathrm{Vs}$, five times smaller than that of CNT/OFET. The current on/off ratio of CNT/OFET device is $5 \times 10^{3}$, whereas $I_{\text {on }} / I_{\text {off }}$ of Au/OFET is only $9 \times 10^{1}$. The current on-off ratio of CNT/OFET device is more than two order magnitude higher than the on-off ratio of Au/OFET. From these results, we conclude that OFET with aligned CNT array electrode shows superior device performance including 

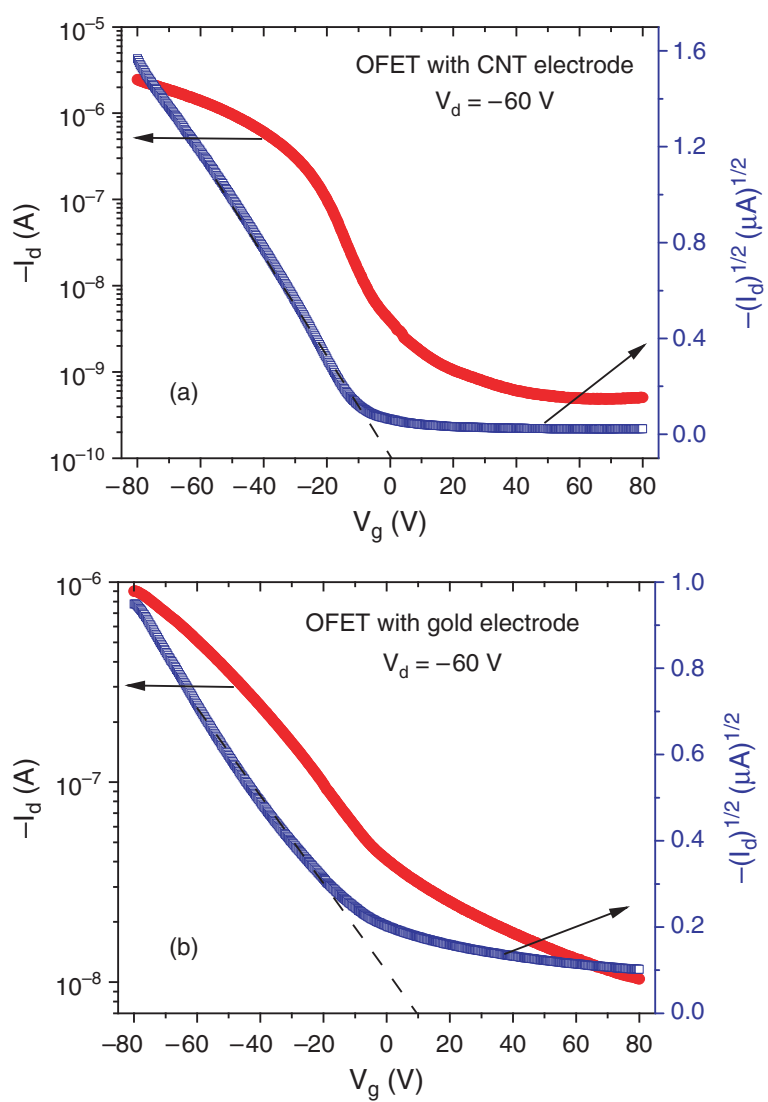

Fig. 4. Transfer characteristics $\left(I_{\mathrm{d}}\right.$ vs. $\left.V_{\mathrm{g}}\right)$ at $V_{\mathrm{d}}=-60 \mathrm{~V}$ (left axis) and $\sqrt{ } I_{\mathrm{d}}$ (right axis) of the P3HT film OFET for (a) CNT aligned array electrode and (b) gold electrode.

higher mobility, higher on-current and higher current on-off ratio than OFET with gold electrodes.

The reasons for better device performance with aligned array CNT electrode compared to Au electrode are as follows: The work function of gold $(5.1 \mathrm{eV})$ and CNT $(5.0 \mathrm{eV})$ are very close, and according to Schottky-Mott model, good contact of P3HT is expected with both gold and CNT. ${ }^{1,6,16}$ However, when gold electrode is contacted with organic material, the dipoles barriers are formed at the interface $e^{6,7}$ and charge carrier transport though the interface is limited by this barrier. In addition, the gold contact exhibits 2D electrostatic effect and it has little influence on the tunneling of the charge carrier though the $\mathrm{Au} / \mathrm{semiconductor}$ interface. On the other hand, the individual CNTs in the CNT array electrodes having a form factor which enhance the electric field at the apex of nanotube that assists the tunneling the charge carrier though the contact barrier. ${ }^{15,16,19}$ Moreover, the strong $\pi-\pi$ bonding between CNT and organic material makes strong interfacial contact and reduces the barrier height. ${ }^{14,16}$

In summary, we have fabricated aligned array carbon nanotube electrodes for organic electronics device. Dielectrophoresis (DEP) technique was used to align the CNT from high quality aqueous solution of CNT with a very high linear density. The CNT source and drain electrodes are fabricated with electron beam lithography followed by etching CNTs with oxygen plasma. We fabricated OFET using both CNT array electrode and gold electrode in order to examine the functionality of the CNT array electrodes. It was found that OFET device with CNT electrode has shown better performance than the device with gold electrode. The improved device performance using CNT array electrode is attributed to absence of dipoles formation, strong bonding interaction between CNT/organic material and the geometry structure of aligned array CNT electrode.

Acknowledgments: This work is partially supported by U.S. National Science Foundation under Grant No. ECCS 0801924 and 1102228.

\section{References and Notes}

1. G. Horowitz, J. Mater. Res. 19, 1946 (2004).

2. C. D. Dimitrakopoulos and P. R. L. Malenfant, Adv. Mater. 14, 99 (2002).

3. C. Di, Y. Liu, G. Yu, and D. Zhu, Account. of Chem. Res. 42, 1573 (2009).

4. S. D. Wang, T. Minari, T. Miyadera, K. Tsukagoshi, and Y. Aoyagi, Appl. Phys. Lett. 91, 203508 (2007).

5. R. Schroeder, L. A. Majewski, and M. Grell, Appl. Phys. Lett. 84, 1004 (2004).

6. N. Koch, A. Kahn, J. Ghijsen, J.-J. Pireaux, J. Schwartz, R. L. Johnson, and A. Elschner, Appl. Phys. Lett. 82, 70 (2003).

7. I. G. Hill, A. Rajagopal, A. Kahn, and Y. Hu, Appl. Phys. Lett. 73, 662 (1998).

8. L. Bürgi, T. J. Richards, R. H. Friend, and H. Sirringhaus, J. Appl. Phys. 94, 6129 (2003).

9. K. A. Singh, G. Sauve, R. Zhang, T. Kowalewski, R. D. McCullough, and L. M. Porter, Appl. Phys. Lett. 92, 263303 (2008).

10. X. Guo, S. Xiaob, M. Myersb, Q. Miaoc, M. L. Steigerwald, and C. Nuckolls, PNAS 106, 691 (2009).

11. X. Guo and C. Nuckolls, J. Mater. Chem. 19, 5470 (2009).

12. P. Qi, A. Javey, M. Rolandi, Q. Wang, E. Yenilmez, and H. Dai, J. Am. Chem. Soc. 126, 11774 (2004).

13. K. Tsukagoshi, I. Yagi, and Y. Aoyagi, Appl. Phys. Lett. 85, 1021 (2004).

14. C. H. Chang, C. H. Chien, and J. Y. Yang, Appl. Phys. Lett. 91, 083502 (2007).

15. F. Cicoira, C. M. Aguirre, and R. Martel, ACS Nano. 5, 283 (2011).

16. C. M. Aguirre, C. Ternon, M. Paillet, P. Desjardins, and R. Martel, Nano Lett. 9, 1457 (2009).

17. A. Southard, V. Sangwan, J. Cheng, E. D. Williams, and M. S. Fuhrer, Org. Electron. 10, 1556 (2009).

18. Y. Y. Zhang, Y. Shi, F. Chen, S. G. Mhaisalkar, L. J. Li, B. S. Ong, and Y. Wu, Appl. Phys. Lett. 91, 223512 (2007).

19. S. L. Hellstrom, R. Z. Jin, R. M. Stoltenberg, and Z. Bao, Adv. Mater. 22, 4204 (2010).

20. S. Shekhar, P. Stokes, and S. I. Khondaker, ACS Nano 5, 1739 (2011).

21. D. Joung, A. Chunder, L. Zhai, S. I. Khondaker, Nanotechnology 21, 165202 (2010).

22. P. Stokes and S. I. Khondaker, Nanotechnology 17, 175202 (2008).

23. P. Stokes and S. I. Khondaker, Appl. Phys. Lett. 96, 083110 (2010). 
24. S. I. Khondaker, IEE Proc.: Circuits Devices and System (special issue in nanoelectronics) 151, 457 (2004).

25. S. I. Khondaker, K. Luo, and Z. Yao, Nanotechnology 21, 095204 (2010).

26. A. Javey, J. Guo, Q. Wang, M. Lundstrom, and H. Dai, Nature 424, 654 (2003).

27. Brewer Science Inc. http://www.brewerscience.com/products/carbonnanotube/.

28. M. Dimaki and P. Boggild, Nanotechnology 15, 1095 (2004)
29. D. Lei, L. Y. Zeng, W. B. Wang, and J. Q. Liang, J. Appl. Phys. 102, 114503 (2007).

30. H. A. Becerril, R. M. Stoltenberg, M. L. Tang, M. E. Roberts, Z. Liu, Y. Chen, D. H. Kim, B.-L. Lee, and Z. Bao, ACS Nano 4, 11 (2010).

31. T. Hirose, T. Nagase, T. Kobayashi, R. Ueda, A. Otomo, and H. Naito, Appl. Phys. Lett. 97, 083301 (2010).

32. J. Zaumseil and H. Sirringhaus, Chem. Rev. 107, 1296 (2007).

Received: 18 January 2011. Revised/Accepted: 22 February 2011. 